\title{
Gasification Pathways and Reaction Mechanisms of Primary Alcohols in Supercritical Water
}

Received 00th October 2019 Accepted 00th January 20xx

DOI: $10.1039 / \times 0 \times x 00000 x$

\author{
Brian R. Pinkard*a, John C. Kramlich ${ }^{a}$, and Igor V. Novosselov ${ }^{a, b}$
}

\begin{abstract}
Supercritical water gasification is a promising waste-to-energy technology with the ability to convert aqueous and/or heterogeneous organic feedstocks to high-value gaseous products, e.g., green hydrogen. Reaction behavior of complex molecules in supercritical water can be inferred through knowledge of the reaction pathways of model compounds in supercritical water. In this study methanol, ethanol, and isopropyl alcohol are gasified in a continuous supercritical water reactor at temperatures between 500 and $560^{\circ} \mathrm{C}$, and for residence times between 3 and $8 \mathrm{~s}$. In situ Raman spectroscopy is used to rapidly identify and quantify reaction products. The experiments confirm the dominance of chain-branching, free radical reaction mechanisms that are responsible for decomposing primary alcohols in the supercritical water environment. Reaction pathways and mechanisms for three alcohols are proposed, conversion metrics are presented, and results are compared with known reaction mechanisms for methanol and ethanol oxidation.
\end{abstract}

\section{Introduction}

Greenhouse gas emissions from the combustion of fossil fuels have stimulated governments and private industry to explore alternative sources and methods for producing liquid transportation fuels. Renewable transportation fuels, such as methanol, ethanol, or biodiesel, have seen limited success in displacing conventional fuels due to the high cost of processing bio-feedstocks ${ }^{1}$, issues of food vs. fuel (bioethanol) ${ }^{2}$, and the compatibility of biofuels with existing combustion platforms ${ }^{3}$. For example, bio-oil and biocrude are chemically unstable and contain high quantities of oxygen, which leads to higher amounts of engine corrosion and poor combustion characteristics. Hydrocracking or hydrotreating, (upgrading of liquid fuels in a hydrogen-rich atmosphere), is necessary to convert most heavy oils or raw biofuels into suitable (drop-in) liquid transportation fuel ${ }^{3,4}$.

Currently, $95 \%$ of industrial hydrogen consumed in the United States is produced via steam reforming of natural gas ${ }^{5}$. One strategy to reduce greenhouse gas emissions is to produce hydrogen from renewable sources. Supercritical water gasification (SCWG) offers a green process for producing renewable hydrogen from organic (carbon-containing) feedstocks. SCWG is particularly attractive as a means of producing hydrogen because heterogeneous and/or aqueous wastes (e.g., sewage sludge, biomass residues, plastics) are

\footnotetext{
a. University of Washington, Mechanical Engineering Department, 3900 E Stevens Way, Seattle, WA 98195

b. University of Washington, Institute for Nanoengineered Systems, Seattle, WA 98195

Electronic Supplementary Information (ESI) available: plots of product formation and decomposition at all tested temperatures, representative Raman spectra of decomposition products, table of Raman peak locations for each relevant compound, experimental Raman spectra as .csv files. See DOI: 10.1039/x0xx00000x
}

suitable feedstocks. However, a better understanding of chemical reactions in supercritical water is needed for successful implementation at the industrial scale.

\section{Supercritical Water Gasification}

Supercritical water (SCW) continues to gain relevance as a green reaction medium for gasification and/or oxidation of organic compounds. As water is heated and pressurized past its critical point $\left(374^{\circ} \mathrm{C}, 22.1 \mathrm{MPa}\right)$, the $\mathrm{H}$-bond network begins to break apart and the dielectric constant decreases, resulting in high solubility of simple and complex organic molecules in $\mathrm{SCW}^{6}$. High temperatures accelerate the breakdown of most organic molecules, either via endothermic gasification pathways, which yield $\mathrm{H}_{2}, \mathrm{CO}, \mathrm{CO}_{2}$, and $\mathrm{CH}_{4}$ or via exothermic oxidation pathways that yield $\mathrm{H}_{2} \mathrm{O}$ and $\mathrm{CO}_{2}{ }^{7}$. Near the critical point, high quantities of $\mathrm{H}^{+}$and $\mathrm{OH}^{-}$ions create an environment favorable for ionic chemistry pathways. At temperatures well above the critical point, the low-density of SCW is an ideal medium for rapid pyrolysis, hydrolysis, and free-radical reaction mechanisms ${ }^{8}$. SCW has been studied as a viable reaction medium to produce gaseous fuel from biomass, sewage sludge, plastics, and agricultural residues ${ }^{6,9}$.

The gaseous product from SCWG (syngas) is rich in $\mathrm{H}_{2}$ with varied yields of $\mathrm{CO}$ and $\mathrm{CH}_{4}$. Key reactions determining the final gaseous yields are the water-gas shift (WGS) reaction:

$$
\mathrm{CO}+\mathrm{H}_{2} \mathrm{O} \leftrightarrow \mathrm{CO}_{2}+\mathrm{H}_{2}
$$

and methanation reactions:

$$
\begin{gathered}
\mathrm{CO}+3 \mathrm{H}_{2} \leftrightarrow \mathrm{CH}_{4}+\mathrm{H}_{2} \mathrm{O} \\
\mathrm{CO}_{2}+4 \mathrm{H}_{2} \leftrightarrow \mathrm{CH}_{4}+2 \mathrm{H}_{2} \mathrm{O}
\end{gathered}
$$


Methanation reactions are exothermic, thus higher temperatures minimize methane production.

Many technical barriers still hinder industrial-scale SCWG. For example, salts in heterogeneous feedstocks precipitate in SCW and corrode or foul reactor components. Char formation is also common, especially when high concentrations of aromatic compounds are present. Engineering solutions to these problems are feasible; however, the knowledge of reaction mechanisms and physical transport properties of reagents at such high temperatures and pressures are required. Basic knowledge of these fundamental phenomena is a first step towards designing robust SCWG reactors for industrialscale waste treatment and hydrogen production.

\section{Batch vs. Continuous Reactors}

The study of reaction chemistry in continuous supercritical water reactors (SCWRs) is of particular interest. Many past studies of model alcohol decomposition in SCW have been conducted in non-reactive, quartz batch reactors ${ }^{10,}{ }^{11}$. While experiments in batch reactors yield important scientific insights, they are of limited practical relevance, as batch reactors have different catalytic and mass transfer behavior than continuous reactors ${ }^{11}$, 12 . Continuous operation is preferred for an industrial-scale system in order to maximize process throughput and yields. Briefly, most continuous reactors are constructed from nickel-base alloys, which are catalytic to gasification reactions. Flow-through operation in tubular reactors increases the interaction of reactants with catalytic reactor walls, especially when the flow regime is turbulent. Finally, reactants in a batch system suffer from slow heating to reaction temperatures, which creates challenges in interpreting chemical reaction behavior.

Studies of reaction chemistry in continuous reactors allows for (i) simulating reaction behavior in practical systems, (ii) in situ data collection for rapidly conducting experiments at short or long residence times ${ }^{13}$, and (iii) near-instantaneous reactant heating and mixing using post-critical injection to achieve a clear reaction starting point ${ }^{14}$. However, it must be noted that inert wall batch reactors offer an opportunity to characterize SCWG chemistry in the absence of a catalytic surface; these are useful benchmarks for understanding the influence of catalysis on the reactions.

\section{SCWG of Model Alcohols}

According to Chakinala et al. ${ }^{15}$, ethanol and methanol are stable in SCW at temperatures up to $600{ }^{\circ} \mathrm{C}$ in the absence of a catalyst. In a study using a batch autoclave reactor, ethanol is reported to produce the highest yield of gaseous products among all alcohols, followed by methanol. All higher chain alcohols were reported to yield lower quantities of gas due to the formation of refractory liquid products. Broadly, all previous studies of primary alcohol gasification in SCW have found that higher temperatures and longer residence times increase conversion to gaseous products, while variations in pressure produce no measurable effect.
Methanol Gasification. Several studies have investigated methanol decomposition in SCW. Boukis et al. ${ }^{16}$ reformed methanol in an Inconel 625 continuous, tubular SCWR at 400 to $600{ }^{\circ} \mathrm{C}$, residence times from 3 to $100 \mathrm{~s}$, and initial methanol loadings from 5 to $64 \mathrm{wt} \% . \mathrm{H}_{2}, \mathrm{CO}, \mathrm{CO}_{2}$, and trace amount of $\mathrm{CH}_{4}$ were detected in the gaseous product. Bennekom et al. ${ }^{17}$ gasified methanol in a continuous reactor at temperatures between 450 and $650{ }^{\circ} \mathrm{C}$ for residence times between 6 and 173 s. Yields of $\mathrm{H}_{2}, \mathrm{CO}$, and $\mathrm{CO}_{2}$ were observed. Trace $\mathrm{CH}_{4}$ yields were reported. Analysis of liquid products revealed trace yields of formaldehyde and formic acid. It was hypothesized that both existed as short-lived reaction intermediates, which is consistent with previous research demonstrating formic acid as an intermediate of the WGS reaction ${ }^{13,18}$.

DiLeo and Savage ${ }^{10}$ investigated the role of nickel as a catalyst for methanol gasification in SCW. The presence of a nickel wire in a quartz batch reactor increased conversion from $20 \%$ after $2 \mathrm{~h}$ to $90 \%$ after $5 \mathrm{~min}$ at $550{ }^{\circ} \mathrm{C}$. It should be noted that nickel catalysis in a continuous reactor is even more significant, as the catalytic effect in the batch setup is limited by the diffusion rate of methanol molecules. $\mathrm{H}_{2}, \mathrm{CO}$, and $\mathrm{CO}_{2}$ were the only products consistently detected in the gaseous phase.

Chakinala et al. ${ }^{15}$ proposed that methanol can decompose to gaseous products in $\mathrm{SCW}$ primarily through $\mathrm{C}-\mathrm{H}$ bond scission to a hydroxymethyl radical $\left(\mathrm{CH}_{2} \mathrm{OH}\right)$ or $\mathrm{O}-\mathrm{H}$ bond scission to a methoxy radical $\left(\mathrm{CH}_{3} \mathrm{O}\right)$ followed by loss of an additional $\mathrm{H}$ to reach formaldehyde $\left(\mathrm{CH}_{2} \mathrm{O}\right)$. Formaldehyde was proposed to decompose to $\mathrm{CO}$ and $\mathrm{H}_{2}$ or to reach formic acid $(\mathrm{HCOOH})$ via oxidation with an $\mathrm{OH}$ radical. A minor pathway to methane via the formation of a methyl $\left(\mathrm{CH}_{3}\right)$ radical by $\mathrm{C}-\mathrm{O}$ bond scission is also proposed.

Ethanol Gasification. Schanzenbächer et al. ${ }^{19}$ gasified ethanol in a continuous SCWR at temperatures from 433 to $494{ }^{\circ} \mathrm{C}$, constant pressure of $24.6 \mathrm{MPa}$, and residence times from 2 to 12 s. Maximum conversion was reported as $16.5 \%$; only acetaldehyde $\left(\mathrm{C}_{2} \mathrm{H}_{5} \mathrm{O}\right)$ was identified as a reaction product.

Arita et al. ${ }^{20}$ studied non-catalytic reaction pathways of ethanol in a batch SCWR. Temperatures from 450 to $500{ }^{\circ} \mathrm{C}$ were maintained for 10 to 60 minutes, with primary reaction products identified as $\mathrm{H}_{2}, \mathrm{CH}_{4}$, and $\mathrm{CO}_{2}$, and minor yields of $\mathrm{CO}$, acetaldehyde, ethylene, and ethane. Two competing reaction pathways were proposed: (i) dehydrogenation of ethanol to acetaldehyde followed by acetaldehyde decomposition to $\mathrm{CO}$ and $\mathrm{CH}_{4}$, or (ii) dehydration of ethanol to ethylene followed by hydrogenation of ethylene to ethane. Global reactions for the two pathways are as follows:

$$
\begin{gathered}
\mathrm{C}_{2} \mathrm{H}_{5} \mathrm{OH} \rightarrow \mathrm{C}_{2} \mathrm{H}_{4} \mathrm{O}+\mathrm{H}_{2} \\
\mathrm{C}_{2} \mathrm{H}_{4} \mathrm{O} \rightarrow \mathrm{CH}_{4}+\mathrm{CO} \\
\mathrm{CO}+\mathrm{H}_{2} \mathrm{O} \rightarrow \mathrm{CO}_{2}+\mathrm{H}_{2} \\
\mathrm{C}_{2} \mathrm{H}_{5} \mathrm{OH} \rightarrow \mathrm{C}_{2} \mathrm{H}_{4}+\mathrm{H}_{2} \mathrm{O} \\
\mathrm{C}_{2} \mathrm{H}_{4}+\mathrm{H}_{2} \rightarrow \mathrm{C}_{2} \mathrm{H}_{6}
\end{gathered}
$$


Chakinala et al. ${ }^{15}$ hypothesized ethanol decomposition pathways via batch reactor studies. Like methanol, $\mathrm{O}-\mathrm{H}$ or $\mathrm{C}-\mathrm{H}$ bond scission is theorized as the dominant mechanism to produce acetaldehyde. At various points in the proposed reaction network, the $\mathrm{C}-\mathrm{C}$ bond can be broken, forming a methyl radical, which ultimately forms $\mathrm{CH}_{4}$. The proposed network is based on observed yields of $\mathrm{H}_{2}, \mathrm{CO}, \mathrm{CO}_{2}, \mathrm{CH}_{4}$, and ethane.

Isopropyl Alcohol Gasification. Previous work on SCWG of 1- or 2propanol is limited. Antal Jr., Carlsson, and $\mathrm{Xu}^{21}$ report yields of propene (C3H6) and 1-propanol after acid-catalyzed dehydration of 2-propanol in subcritical water at $34.5 \mathrm{MPa}$ and $320^{\circ} \mathrm{C}$ for residence times up to $100 \mathrm{~s}$. Chakinala et al. ${ }^{15}$ gasified 1-propanol in SCW, noting trace yields of benzene and toluene. The postulated reaction network again includes initial $\mathrm{C}-\mathrm{H}$ and $\mathrm{O}-\mathrm{H}$ bond scission steps, leading to acetone $\left(\mathrm{C}_{3} \mathrm{H}_{6} \mathrm{O}\right)$, which is thought to break down to $\mathrm{CH}_{4}$ and $\mathrm{CO}$. Other theorized steps include reactions to form ethylene, ethane, and various intermediate products.

\section{Reaction Mechanisms and Pathways}

The experiments conducted in this study are meant to fully elucidate the dominant reaction mechanisms involved in primary alcohol decomposition in SCW. While previous studies have roughly defined the reaction networks, many discrepancies exist, and the conditions necessary for full conversion of alcohols in a continuous reactor are still largely unknown. For practical relevance, a continuous tubular reactor is used, and in situ Raman spectroscopy allows for collected product yield data after reactions of short residence times. Ultimately, insights are sought into SCWG chemistry for furthering the understanding of this green reaction medium towards industrial-scale implementation.

\section{Materials and Methods}

A continuous SCWG reactor with in situ Raman spectroscopy is used to perform all experiments. Pure methanol, ethanol, or IPA were continuously introduced into the SCWG reactor at an overall volumetric loading of 10 vol\%, corresponding to 8.09 wt\%, $8.06 \mathrm{wt} \%$, and $8.03 \mathrm{wt} \%$ initial respective mass fractions. All reagents were used as received without further purification. The reactor design and design methodology have been described elsewhere ${ }^{12,}{ }^{13}$. Briefly, a custom mixing section is used to inject cold reagent into a bulk flow of supercritical deionized water, at a volumetric flow ratio selected to achieve rapid mixing and heating as detailed in Tiwari et al. ${ }^{14}$. Postcritical injection achieves a well-defined reaction initiation point, and a heat exchanger rapidly quenches products after a pre-defined residence time. All reactants and products exist in a well-mixed, supercritical fluid phase at reaction conditions.

Raman spectra are collected for each experimental condition, using a flow-through optical cell and a fiber-optic immersion Raman probe operating in the backscatter configuration $^{22}$. Spectra are translated both to qualitative (product identification) and quantitative (molecular concentrations) data relevant to understanding reaction pathways and rates. Indirect hard modeling (IHM) is used for accurate quantitative Raman spectroscopy for calculating product yields ${ }^{23}, 24$. PEAXACT spectral processing software is used to perform IHM. Quantitative calibration of Raman spectra was achieved via an indirect methodology closely aligned with that described by Beumers et al. ${ }^{25}$ where an elemental balance of reactor inputs and outputs is used to achieve calibration.

Due to the inherent challenge of repeatedly performing SCWG experiments, replicate experiments were not performed. Instead, five replicate Raman spectra were captured for each experimental condition, thus the error measurements presented on all figures represent an overall error in data collection and processing, not experimental error. Gasification efficiency, the ratio of the mass fraction of the gaseous products to the mass fraction of the reagent, is used as a key metric to quantify the efficacy of the SCWG process at each tested condition.

\section{Results}

Methanol

SCWG of methanol primarily yields $\mathrm{H}_{2}$ and $\mathrm{CO}$, with secondary yields of $\mathrm{CO}_{2}$ and trace formaldehyde production, as shown in Figure 1. These profiles illustrate the sequential nature of product formation; $\mathrm{H}_{2}$ is detectable at $4 \mathrm{~s}$, followed by $\mathrm{CO}$ at $5 \mathrm{~s}$, and $\mathrm{CO}_{2}$ at $7 \mathrm{~s}$. Formaldehyde is confirmed as a short-lived reaction intermediate; however, no yields of formic acid or methane are detected. We propose the reaction network in Figure 2, with methanol dehydrogenating to formaldehyde, followed by decomposition to $\mathrm{CO}$ and $\mathrm{H}_{2}$.

Our results indicate that the reaction mechanisms hypothesized by Chakinala et al. ${ }^{15}$ to generate formic acid (formaldehyde oxidation) or methane (methyl radical generation) are unlikely to be active during SCWG. Trace methane yields have been reported ${ }^{16,17}$, but it is more likely

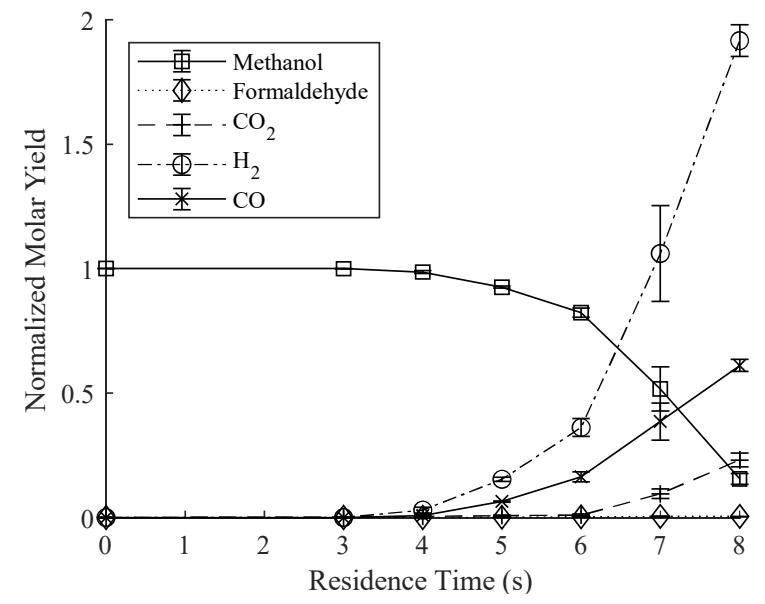

Figure 1: Formation and decomposition of reaction products during SCWG of methanol at $560^{\circ} \mathrm{C}$ 


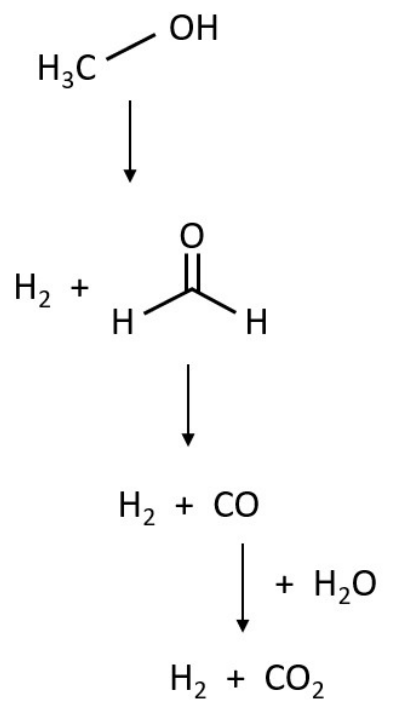

Figure 2: Methanol decomposition network in supercritical water

that secondary methanation reactions are responsible for this observation.

The WGS reaction is responsible for the observed maximum $\mathrm{CO}_{2}$ yield of $0.23 \mathrm{~mol}-\mathrm{CO}_{2} / \mathrm{mol}-\mathrm{MeOH}$, but low yields indicate that the WGS reaction does not have sufficient residence time to reach completion. Full conversion of methanol along the network in Figure 2 would result in a maximum of $1 \mathrm{~mol}-$ $\mathrm{CO}_{2} / \mathrm{mol}-\mathrm{MeOH}$ and $3 \mathrm{~mol}-\mathrm{H}_{2} / \mathrm{mol}-\mathrm{MeOH}$. Full conversion of $\mathrm{CO}$ would increase $\mathrm{H}_{2}$ yields past the observed maximum of 1.92 $\mathrm{mol}-\mathrm{H}_{2} / \mathrm{mol}-\mathrm{MeOH}$. Gasification efficiency (GE) is plotted against residence time for all tested temperatures in Figure 3. GE approaches values above $100 \%$ at $560{ }^{\circ} \mathrm{C}$, due to the conversion of liquid $\mathrm{H}_{2} \mathrm{O}$ to gaseous $\mathrm{H}_{2}$ via the WGS. No other methanol decomposition products are detected over the tested temperature and residence time range, and reaction profiles at all temperatures follow similar trends. Plots of compound formation and decomposition at all tested temperatures are available in Figure 12 (supplementary information), and a

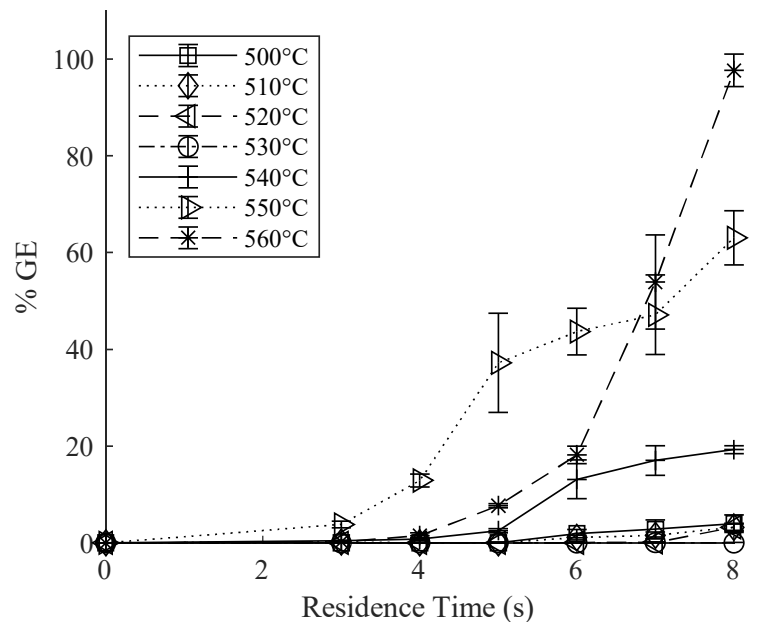

Figure 3: Gasification Efficiency of methanol at all tested temperatures

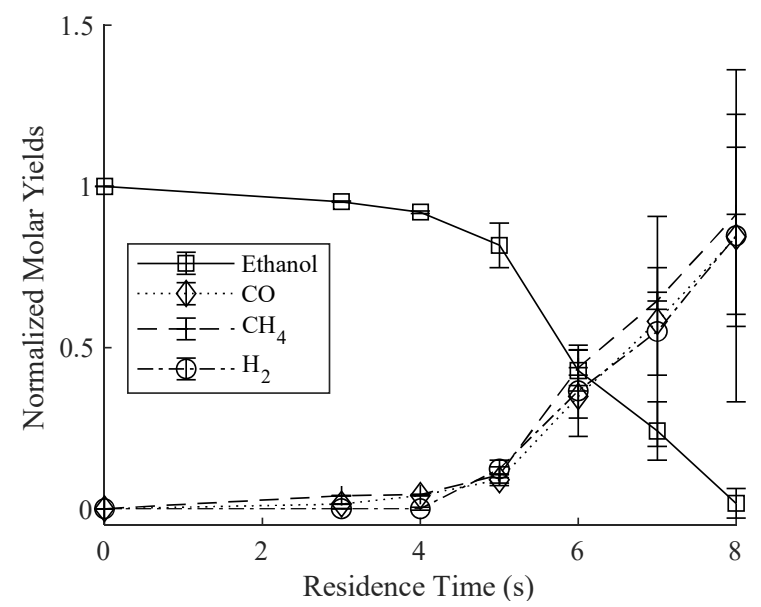

Figure 4: Formation and decomposition of major reaction products during SCWG of ethanol at $560^{\circ} \mathrm{C}$

representative Raman spectrum of methanol gasification products is shown in Figure 16 (supplementary information).

\section{Ethanol}

Acetaldehyde and ethylene are the first observable products during the SCWG of ethanol, as shown at $3 \mathrm{~s}$ in Figure 5. This is followed by a significant increase in $\mathrm{H}_{2}, \mathrm{CO}$, and $\mathrm{CH}_{4}$ yields at $5 \mathrm{~s}$, as seen in Figure 4. Acetaldehyde yields continue to increase to a maximum of 0.12 mol- $\mathrm{C}_{2} \mathrm{H}_{4} \mathrm{O} / \mathrm{mol}-\mathrm{EtOH}$ at $6 \mathrm{~s}$, followed by a similarly-paced decrease to $0.005 \mathrm{~mol}$ $\mathrm{C}_{2} \mathrm{H}_{4} \mathrm{O} / \mathrm{mol}-\mathrm{EtOH}$, confirming its role as a short-lived intermediate. Once sufficient $\mathrm{H}_{2}$ is generated for ethylene hydrogenation, ethane emerges as a detectable product at $6 \mathrm{~s}$. Finally, a $\mathrm{CO}_{2}$ yield of $0.04 \mathrm{~mol}-\mathrm{CO}_{2} / \mathrm{mol}-\mathrm{EtOH}$ is measured at 8 $\mathrm{s}$, again resulting from the WGS reaction. Figure 4 demonstrates that $\mathrm{H}_{2}, \mathrm{CO}$, and $\mathrm{CH}_{4}$ are produced in nearly equimolar quantities, reaching maximum respective yields of $0.85 \mathrm{~mol}-$ $\mathrm{H}_{2} / \mathrm{mol}-\mathrm{EtOH}, 0.84 \mathrm{~mol}-\mathrm{CO} / \mathrm{mol}-\mathrm{EtOH}$ and $0.91 \mathrm{~mol}-\mathrm{CH}_{4} / \mathrm{mol}-$ $\mathrm{EtOH}$. This supports the hypothesis that acetaldehyde

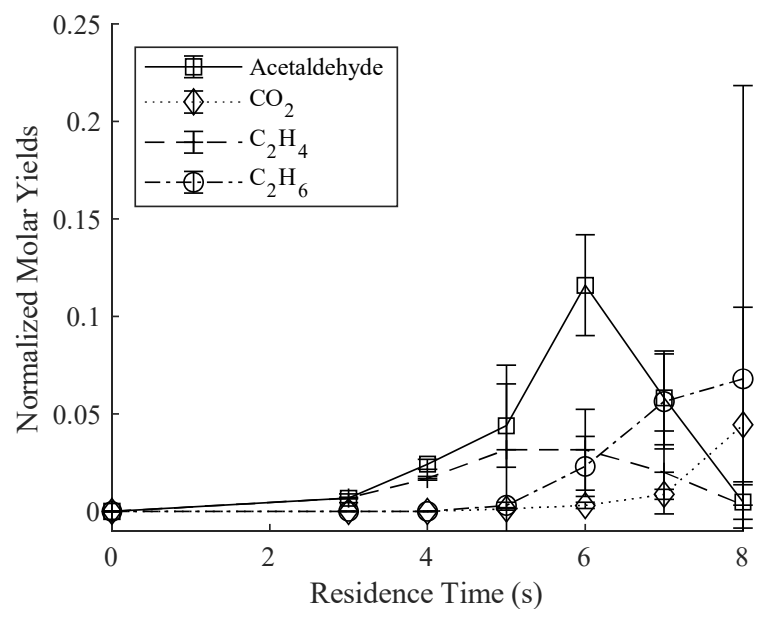

Figure 5: Formation and Decomposition of minor reaction products during SCWG of ethanol at $560^{\circ} \mathrm{C}$ 


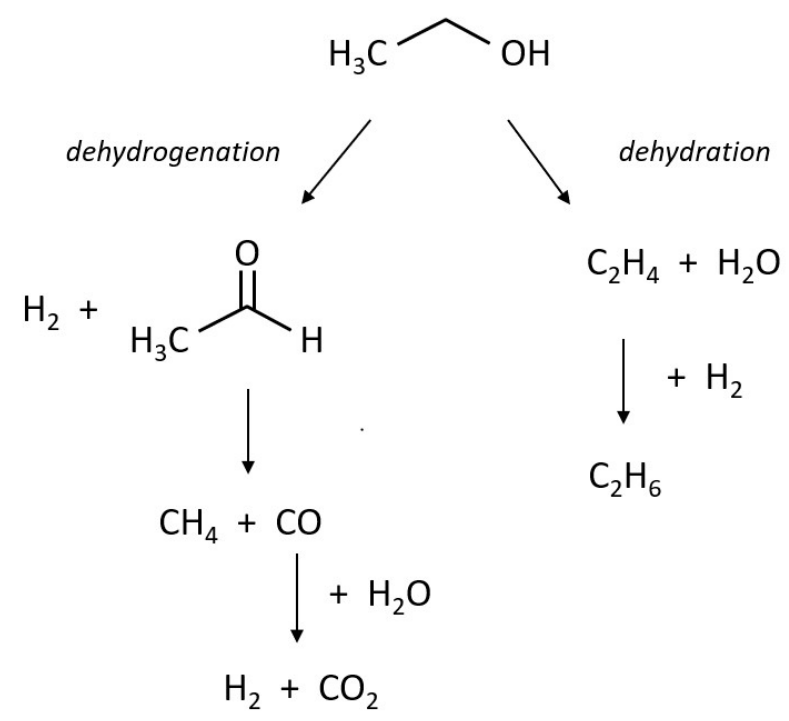

Figure 6: Ethanol decomposition network in supercritical water

decomposition is responsible for the formation of $\mathrm{CO}$ and $\mathrm{CH}_{4}$, with molar $\mathrm{CO}$ yields slightly lower due to consumption via the WGS. The trends described here are also observed during SCWG of ethanol at other tested temperatures; these formation and decomposition profiles are available in Figures 13 and 14 (supplementary information).

Interpreting the observed product yields, ethanol decomposition follows the reaction pathways illustrated in Figure 6, confirming the global reactions proposed by Arita et al. ${ }^{20}$. Two competing reaction pathways are active, the primary being dehydrogenation to acetaldehyde, with a secondary pathway of dehydration to ethylene. Acetaldehyde is rapidly converted to $\mathrm{CO}$ and $\mathrm{CH}_{4}$, while ethylene can hydrogenate to ethane if suitable $\mathrm{H}_{2}$ is present. Gasification efficiency is plotted against residence time for all tested temperatures in Figure 7. A representative Raman spectrum showing reaction products is available in Figure 18 (supplementary information).

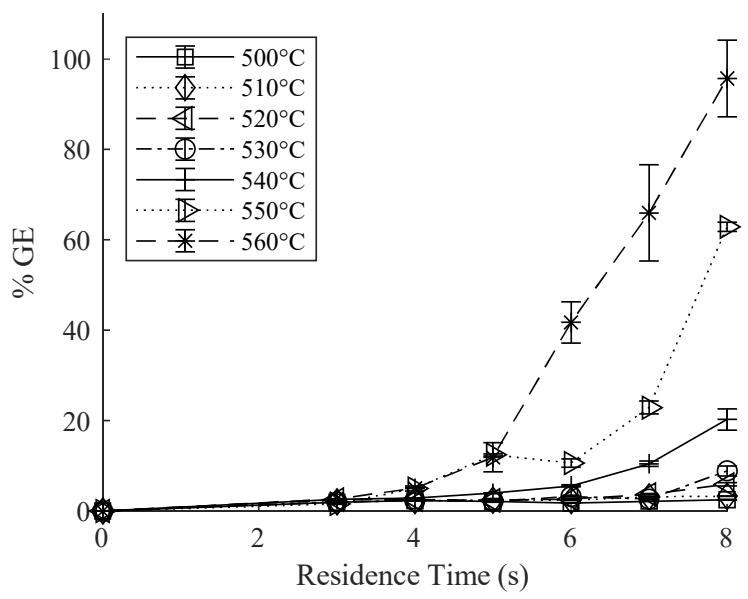

Figure 7: Gasification Efficiency of ethanol at all tested temperatures

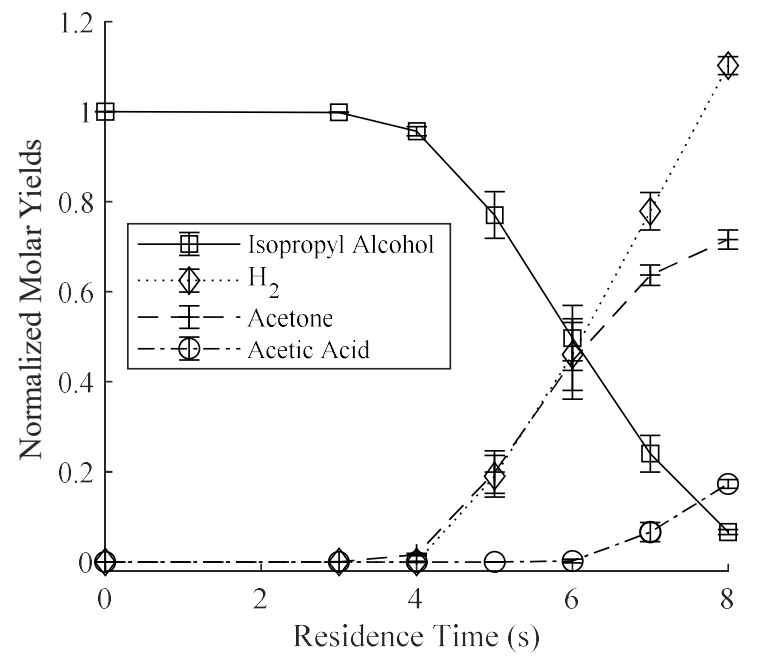

Figure 8: Formation and decomposition of major reaction products during SCWG of IPA at $560^{\circ} \mathrm{C}$

\section{Isopropyl Alcohol}

Production of $\mathrm{H}_{2}$ and acetone begin simultaneously and in nearly equimolar quantities from $4-6 \mathrm{~s}$, as shown in Figure 8. Acetone can subsequently decompose to several different product species, which leads to its maximum observed yield of 0.72 mol- $\mathrm{C}_{3} \mathrm{H}_{6} \mathrm{O} / \mathrm{mol}-\mathrm{IPA}$. To identify acetone decomposition products, SCWG of acetone was performed at $560^{\circ} \mathrm{C}$ for $8 \mathrm{~s}$. The collected Raman spectrum is available in Figure 20 (supplementary information), from which major acetone gasification products were identified as acetic acid and $\mathrm{CH}_{4}$, with small yields of $\mathrm{H}_{2}, \mathrm{CO}, \mathrm{CO}_{2}$, ethylene, and ethane. The formation of acetic acid at $7 \mathrm{~s}$ in Figure 8 confirms this, as does the minor production of methane in Figure 9. These observed products led to the proposed acetone decomposition pathways presented in Figure 10. Analysis of IPA data shows many similarities with trends from SCWG of methanol and ethanol, such as the delayed appearance of $\mathrm{CO}_{2}$ at $7 \mathrm{~s}$. Only trace

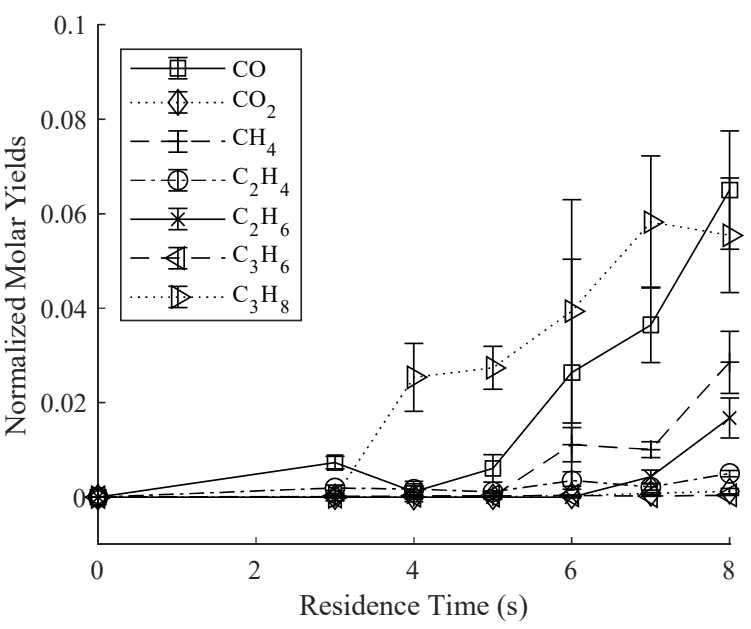

Figure 9: Formation and decomposition of minor reaction products during SCWG of IPA at $560{ }^{\circ} \mathrm{C}$ 


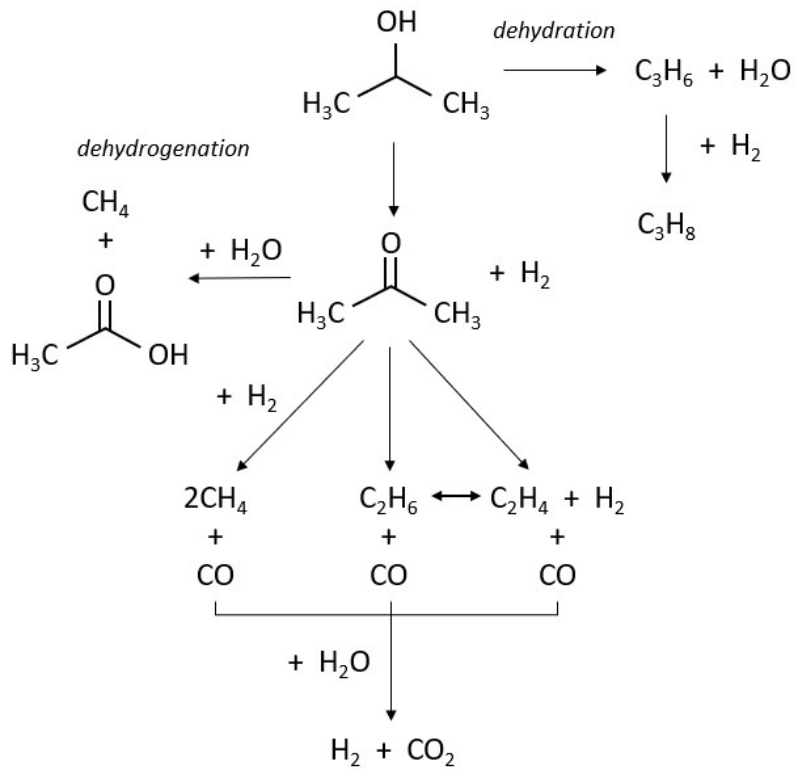

Figure 10: IPA decomposition network in SCW

amounts of $\mathrm{C}_{2} \mathrm{H}_{4}$, and $\mathrm{C}_{2} \mathrm{H}_{6}$ are witnessed indicating that conversion of acetone to these products is not favorable.

Trace propene yields of are detected, which rapidly hydrogenates to a maximum propane yield of $0.06 \mathrm{~mol}$ $\mathrm{C}_{3} \mathrm{H}_{8} /$ mol-IPA at $7 \mathrm{~s}$. Detection of propene and propane confirm the dehydration pathway is active for SCWG of IPA.

Similar to ethanol, we propose that IPA decomposes via competing dehydrogenation and dehydration reaction pathways, as presented in Figure 10. Many of the reaction pathways are inferred from previous knowledge, such as ethylene hydrogenation and the WGS reaction. Acetic acid is a known refractory SCWG product, thus its decomposition to gaseous products is unlikely to be significant.

Gasification efficiency is plotted against residence time for all tested temperatures in Figure 11. GE is drastically lower for SCWG of IPA than methanol or ethanol, due to the formation of

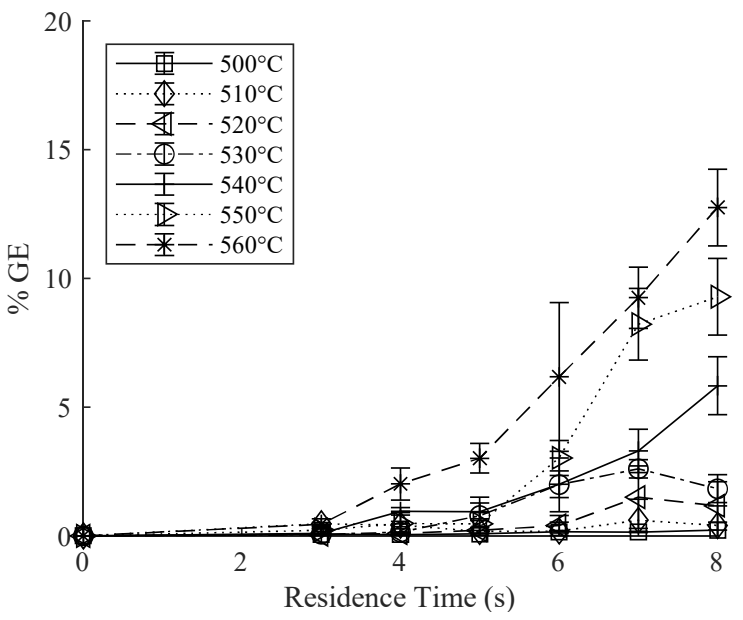

Figure 11: Gasification efficiency of IPA at all tested temperatures acetone and acetic acid in significant quantities. Decomposition and formation profiles of IPA reaction products at all tested temperatures are available in Figure 15 and 16 (supplementary information). A representative Raman spectrum of IPA decomposition products is presented in Figure 19 (supplementary information)

\section{Discussion}

From the decomposition profiles, we infer that the primary mechanisms driving the decomposition of alcohols in SCW are chain-branching, free radical reactions. Based on reaction profiles which consistently show a reaction induction time between 3 and $8 \mathrm{~s}$, we conclude that radical pooling is a key step facilitating alcohol decomposition. The use of time-resolved, in situ Raman spectroscopy led to this observation, which cannot be detected in batch reactor experiments due to the slow heating rate and longer residence times.

Clearly, alcohol decomposition in SCW does not follow firstorder reaction behavior at these conditions; therefore, alcohol decomposition cannot be explained by hydrolysis or pyrolysis alone. $\mathrm{H}$ abstraction is a key mechanism for all three alcohols studied, confirmed by the dominance of dehydrogenation pathways to formaldehyde, acetaldehyde, and acetone. Based on the work of DiLeo and Savage ${ }^{10}$, along with comparison of these results to studies performed in batch reactors ${ }^{15}, 20$, we also propose that $\mathrm{H}$ abstraction and radical generation is promoted by the catalytic reactor walls.

\section{Insights from Alcohol Oxidation Literature}

Previous modeling and experimental work have shown that SCW oxidation chemistry of alcohols is analogous to oxidation chemistry of alcohols under standard combustion conditions ${ }^{26}$. It follows that reactions of alcohols in SCW without an oxidant would follow similar reaction mechanisms as those already described and quantified in the combustion literature involving $\mathrm{H}, \mathrm{OH}$, and other intermediate radicals. It is plausible thatSCWG of alcohols could be modeled using published reaction mechanisms and rates from the combustion literature, provided that the catalytic effect of the reactor wall can be quantified.

An important finding in alcohol combustion studies is that direct abstraction of the $\mathrm{OH}$ radical is a negligible reaction mechanism (e.g., $\left.\mathrm{CH}_{3} \mathrm{OH} \rightarrow \mathrm{CH}_{3}+\mathrm{OH}\right)^{27,28}$. The absence of $\mathrm{CH}_{4}$ as a product from methanol SCWG indicates that this finding holds in SCW environments. This also indicates that ethanol and IPA dehydration must proceed through $\mathrm{H}$ abstraction.

Initial $\mathrm{H}$ abstraction from the methanol molecule can form $\mathrm{CH}_{3} \mathrm{O}$ or $\mathrm{CH}_{2} \mathrm{OH}$ radicals, as proposed by Chakinala et al. ${ }^{15}$. Regardless, both ultimately decompose to formaldehyde through an additional $\mathrm{H}$ abstraction step, generating a pool of $\mathrm{H}$ radicals. Formaldehyde further breaks down quite rapidly to $\mathrm{CO}$ and $\mathrm{H}_{2}$; aldehydes are highly sensitive to radical attack and are short-lived at the conditions tested ${ }^{28}$.

In 1992, Norton and Dryer ${ }^{28}$ concluded that the variation in observed products during ethanol oxidation was largely 
dependent on the initial site of $\mathrm{H}$ abstraction. This finding seems to hold for SCWG of ethanol. Three potential $\mathrm{C}_{2} \mathrm{H}_{5} \mathrm{O}$ isomers are proposed as being energetically accessible: $\mathrm{CH}_{3} \mathrm{CHOH}, \mathrm{C}_{2} \mathrm{H}_{4} \mathrm{OH}$, and $\mathrm{CH}_{3} \mathrm{CH}_{2} \mathrm{O}^{28}, 29$. These isomers can then react via the following reactions:

$$
\begin{gathered}
\mathrm{CH}_{3} \mathrm{CHOH} \rightarrow \mathrm{C}_{2} \mathrm{H}_{4} \mathrm{O}+\mathrm{H} \\
\mathrm{C}_{2} \mathrm{H}_{4} \mathrm{OH} \rightarrow \mathrm{C}_{2} \mathrm{H}_{4}+\mathrm{OH} \\
\mathrm{CH}_{3} \mathrm{CH}_{2} \mathrm{O} \rightarrow \mathrm{CH}_{2} \mathrm{O}+\mathrm{CH}_{3}
\end{gathered}
$$

all of which produce more free radicals in the reaction environment to speed the initial $\mathrm{H}$ abstraction step. No formaldehyde was detected during SCWG of ethanol; it is likely that it decomposes to $\mathrm{CO}$ and $\mathrm{H}_{2}$ too quickly to be detected.

Detailed oxidation mechanisms are not available in the literature for IPA, but it is reasonable to extrapolate dominant mechanisms based on methanol and ethanol mechanisms. The longer carbon chain affords four potential $\mathrm{C}_{3} \mathrm{H}_{7} \mathrm{O}$ isomers after $\mathrm{H}$ abstraction from the parent IPA molecule. If the reaction behavior is similar to ethanol, (and the similarity of the reaction network and decomposition profiles seems to indicate that it is), these isomers could plausibly react via the following reactions:

$$
\begin{gathered}
\mathrm{CH}_{3} \mathrm{CH}_{2} \mathrm{CHOH} \rightarrow \mathrm{C}_{3} \mathrm{H}_{6} \mathrm{O}+\mathrm{H} \\
\mathrm{CH}_{3} \mathrm{CHCH}_{2} \mathrm{OH} \rightarrow \mathrm{C}_{3} \mathrm{H}_{6} \mathrm{O}+\mathrm{H} \\
\mathrm{C}_{3} \mathrm{H}_{6} \mathrm{OH} \rightarrow \mathrm{C}_{3} \mathrm{H}_{6}+\mathrm{OH} \\
\mathrm{CH}_{3} \mathrm{CH}_{2} \mathrm{CH}_{2} \mathrm{O} \rightarrow \mathrm{CH}_{2} \mathrm{O}+\mathrm{C}_{2} \mathrm{H}_{5}
\end{gathered}
$$

Acetone is the dominant intermediate from IPA decomposition. Similar to formaldehyde and acetaldehyde, the $\mathrm{C}=\mathrm{O}$ bond in the acetone molecule is stable. Acetone decomposition in SCW must proceed through $\mathrm{C}-\mathrm{C}$ bond cleavage, forming a methyl radical and a methylcarbonyl radical $\left(\mathrm{C}_{2} \mathrm{H}_{3} \mathrm{O}\right)$, or forming an ethyl radical $\left(\mathrm{C}_{2} \mathrm{H}_{5}\right)$ and formaldehyde. This $\mathrm{C}-\mathrm{C}$ bond cleavage is likely enhanced by the catalytic reactor surface ${ }^{8}$. The methylcarbonyl radical can gain a hydroxyl $(\mathrm{OH})$ radical to form acetic acid, which appears to be the most likely acetone decomposition pathway. If the ethyl radical is formed it could ultimately react to ethylene or ethane, and the formaldehyde would rapidly decompose to $\mathrm{CO}$ and $\mathrm{H}_{2}$. This explains the minor yields of ethane, ethylene, $\mathrm{CO}, \mathrm{H}_{2}$, and $\mathrm{CO}_{2}$ from SCWG of acetone. Because the molar yields of acetic acid are consistently higher than the molar yield of $\mathrm{CH}_{4}$, it seems highly unlikely that acetone can simultaneously lose both methyl radicals along the hydrogenation pathway in Figure 8; however, it is included for completeness as it cannot be directly ruled out.

The most likely site of $\mathrm{H}$ abstraction depends on the bond energies of the various $\mathrm{C}-\mathrm{H}$ and $\mathrm{O}-\mathrm{H}$ bonds in each molecule. For ethanol, the $\mathrm{CH}_{3} \mathrm{CHOH}$ is most energetically accessible, which results in dehydrogenation manifesting as the dominant reaction pathway. Analogously, during IPA decomposition, $\mathrm{CH}_{3} \mathrm{CH}_{2} \mathrm{CHOH}$ is the most energetically accessible isomer after $\mathrm{H}$ abstraction, which would decompose to acetone. Low yields of ethylene, ethane, and no observed formaldehyde yields from IPA decomposition indicate that it is not energetically favorable to reach the $\mathrm{CH}_{3} \mathrm{CH}_{2} \mathrm{CH}_{2} \mathrm{O}$ isomer.

Based on previous experimental work on ethanol pyrolysis at $677{ }^{\circ} \mathrm{C}$, which required $>30 \mathrm{~s}$ to achieve full ethanol decomposition ${ }^{30}$, we speculate that pyrolysis mechanisms are not significant to alcohol decomposition in SCW at the conditions studied.

\section{Char Formation Mechanisms}

The abstraction of $\mathrm{H}$ atoms through $\mathrm{C}-\mathrm{H}$ and $\mathrm{O}-\mathrm{H}$ bond scission is shown to occur readily in SCW, even in the absence of an oxidant. The initial abstraction mechanisms must result from $\mathrm{H}$ and $\mathrm{OH}$ radicals present in $\mathrm{SCW}$ above $500{ }^{\circ} \mathrm{C}$, or from catalytic abstraction. As the radical pool grows, $\mathrm{H}$ abstraction accelerates, leading to the observed chain-branching decomposition profiles. Additionally, open C bonding sites become active. This could explain previously published results, where char yields during SCWG of aromatic compounds or heterogeneous feedstocks are higher than expected ${ }^{31}$.

Analogous behavior occurs during the combustion of hydrocarbons, via the $\mathrm{H}$-abstraction $-\mathrm{C}_{2} \mathrm{H}_{2}$-addition (HACA) mechanism, as first proposed by Frenklach ${ }^{32}$. As $\mathrm{H}$ is abstracted from aromatic compounds, the open bonding sites become occupied by acetylene $\left(\mathrm{C}_{2} \mathrm{H}_{2}\right)$ radicals resulting in polyaromatic hydrocarbon (PAH) growth and soot formation in flames. The HACA regime is dominant under high concentrations of $H$ radicals and low concentrations of $O$ radicals. As demonstrated here, SCWG produces copious $\mathrm{H}$ radicals when hydrocarbons are present. During SCWG of aromatic compounds, char formation likely proceeds via mechanistically similar or identical steps to HACA. This hypothesis must be investigated more thoroughly through a methodological study of char structure and formation mechanisms. However, there exists support for this hypothesis in a recent study by Matsumura et al. ${ }^{33}$, where $\mathrm{H}$ radical scavengers were shown to effectively suppress char formation during SCWG of guaiacol.

\section{Conclusions}

Primary alcohols are gasified in SCW at temperatures between 500 and $560{ }^{\circ} \mathrm{C}$ for residence times between 3 and 8 s. In situ Raman spectroscopy facilitates rapid data collection at short residence times. Data collected at short residence times demonstrate the procession of chain-branching, free radical mechanisms for all primary alcohols. Radical pooling likely occurs through $\mathrm{H}$ abstraction, which is analogous to known alcohol combustion mechanisms and could explain high char yields witnessed during SCWG of aromatic compounds. Mechanistic similarities between SCWG and combustion of alcohols could simplify future modeling efforts. $\mathrm{H}$ abstraction and $\mathrm{C}-\mathrm{C}$ bond scission is likely accelerated by the presence of a catalytic reactor surface, and by the efficient mass transfer 
achieved with a continuous reactor. A more complete understanding of SCWG reaction mechanisms allows for prediction of decomposition pathways of more complex organic compounds, advancing this green technology towards industrial implementation.

\section{Conflicts of interest}

There are no conflicts to declare.

\section{Acknowledgments}

Funding for this work was provided by the Defense Threat Reduction Agency (DTRA) - Grant HDTRA1-17-1-0001. Special thanks to Vedant Maheshwari, Anmol Purohit, and Elizabeth Rasmussen for assistance collecting experimental data, and to David Gorman and Kartik Tiwari for help with the initial design and fabrication of the reactor used for these experiments.

\section{References}

1 M. A., Carriquiry, X. Du, G. R. Timilsina, Energy Policy, 2011 39, 4222

2 A. Gupta, J. Verma, Renew. Sust. Energy. Rev., 2015, 41, 550.

3 A. H. Zacher, M. V. Olarte, D. M. Santosa, D. C. Elliott, S. B. Jones, Green Chemistry, 2014, 16, 491.

4 J. Scherzer, A. J. Gruia, Hydrocracking Science and Technology, Taylor \& Francis, Abingdon, 1996.

5 Hydrogen Production, DOE hydrogen program 2016. Available from:

\{https://www.energy.gov/sites/prod/files/2016/07/f33/fcto _hydrogen_production_fs.pdf\}.

6 A. Kruse, Biofuels Bioprod. Biorefin., 2008, 2, 415.

7 P. E. Savage, Chem. Rev., 1999, 99, 603.

8 Y. Guo, S. Z. Wang, D. H. Xu, Y. M. Gong, H. H. Ma, X. Y. Tang, Ren. Sus. Energ. Rev. 2010, 14, 334.

9 M. J. Antal Jr., S. G. Allen, D. Schulman, X. Xu, R. J. Divilio, Ind. Eng. Chem. Res., 2000, 39, 4040.

10 G.J. DiLeo, and P.E. Savage, J. Supercrit. Fluids, 2006, 39, 228.

11 B. R. Pinkard, D. J. Gorman, K. Tiwari, J. C. Kramlich, P. G. Reinhall, and I. V. Novosselov, Ind. Eng. Chem. Res., 2018, 57, 3471.

12 B. R. Pinkard, D. J. Gorman, K. Tiwari, E. G. Rasmussen, J. C. Kramlich, P. G. Reinhall, and I. V. Novosselov, Heliyon, 2019, 5, e01269.

13 B. R. Pinkard, D. J. Gorman, E. G. Rasmussen, J. C. Kramlich, P. G. Reinhall, and I. V. Novosselov, Int. J. Hydrog. Energy, 2019, In Press.

14 K. Tiwari, B. R. Pinkard, D. J. Gorman, J. Davis, J. C. Kramlich, P. G. Reinhall, and I. V. Novosselov, Proceedings of the $12^{\text {th }}$ International Symposium on Supercritical Fluids, 2018.

15 A. G. Chakinala, S. Kumar, A. Kruse, S. R. A. Kersten, W. P. M. van Swaaij, and D. W. F. Brilman, J. Supercrit. Fluids, 2012, 74, 8.

16 N. Boukis, V. Diem, W. Habicht, E. Dinjus, Ind. Eng. Chem. Res., 2003, 42, 728.

17 J. G. van Bennekom, R. H. Venderbosch, D. Assink, H. J. Heeres, J. Supercrit. Fluids, 2011, 58, 99.

18 K. Yoshida, C. Wakai, N. Matubayasi, M. Nakahara, J. Phys. Chem. A, 2004, 108, 7479.

19 J. Schanzenbächer, J. Taylor, and J. Tester, J. Supercrit. Fluids, 2002, 22, 139
20 T. Arita, K. Nakahara, K. Nagami, and O. Kajimoto, Tetrahedron Lett., 2003, 48, 1083.

21 M. J. Antal, Jr., M. Carlsson, and X. Xu, Ind. Eng. Chem. Res., 1998, 37, 3820.

22 B. R. Pinkard, D. J. Gorman, E. G. Rasmussen, V. Maheshwari, J. C. Kramlich, P. G. Reinhall, I. V. Novosselov, Data in Brief, 2019, In Press.

23 E. Kriesten, F. Alsmeyer, A. Bardow, and W. Marquardt, Chemom. Intell. Lab. Syst. 2008, 91, 181.

24 F. Alsmeyer, H. J. Koss, and W. Marquardt, Appl. Spectrosc. 2004, 58, 975.

25 P. Beumers, T. Brands, H. J. Koss, and A. Bardow, Fluid Phase Equilib., 2016, 424, 52.

26 S. F. Rice, E. Croiset, Ind. Eng. Chem. Res. 2001, 40, 86.

27 T. J. Held, F. L. Dryer, Int. J. Chem. Kin. 1998, 30, 805.

28 T. S. Norton, F. L. Dryer, Int. J. Chem. Kin. 1992, 24, 319.

29 N. M. Marinov, Int. J. Chem. Kin. 1999, 31, 183.

30 J. Li, A. Kazakov, F. L. Dryer, Int. J. Chem. Kin. 2001, 33, 859.

31 C. M. Huelsman and P. E. Savage, Phys. Chem. Chem. Phys., 2012, 14, 2900.

32 M. Frenklach, Phys. Chem. Chem. Phys., 2002, 4, 2028.

33 Y. Matsumura, S. Goto, Y. Takase, S. Inoue, T. Inoue, Y. Kawai, T. Noguchi, H. Tanigawa, Energy Fuels, 2018, 32, 9568.

34 G. Magnotti, U. KC, P. L. Varghese, R. S. Barlow, J. Quant. Spectrosc. Radiat. Transf. 2015, 163, 80. 